\title{
Dynamic hyperinflation and tolerance to interval exercise in patients with advanced COPD
}

\author{
I. Vogiatzis*\#, S. Nanas*, E. Kastanakis ${ }^{\Uparrow}$, O. Georgiadou\#, O. Papazahou*, Ch. Roussos*
}

Dynamic hyperinflation and tolerance to interval exercise in patients with advanced COPD. I. Vogiatzis, S. Nanas, E. Kastanakis, O. Georgiadou, O. Papazahou, Ch. Roussos. C ERS Journals Ltd 2004.

ABSTRACT: Dynamic hyperinflation (DH) contributes importantly to the limitation of constant-load exercise (CLE) in patients with chronic obstructive pulmonary disease (COPD). However, its role in the limitation of interval exercise (IE) remains to be explored.

The change $(\Delta)$ in inspiratory capacity (IC) was measured to reflect changes in DH in 27 COPD patients (forced expiratory volume in one second mean \pm SEM \% predicted: $40 \pm 3$ ) at the end of a symptom-limited CLE test at $80 \%$ of peak work capacity (WRmax) and an IE test at 100\% WRmax $(30 \mathrm{~s}$ of work, alternated with $30 \mathrm{~s}$ of unloaded pedalling).

At the limit of tolerance in both IE and CLE, patients exhibited similar DH ( $\triangle \mathrm{IC}$ : $0.39 \pm 0.05 \mathrm{~L}$ and $0.45 \pm 0.05 \mathrm{~L}$, respectively). However, exercise endurance time (tend) for IE (32.7 $\pm 3.0 \mathrm{~min})$ was significantly greater than for CLE (10.3 $\pm 1.6 \mathrm{~min})$. The IE tend correlated with resting IC, expressed as \% pred normal. At 30 and $90 \%$ of total IE tend, $\Delta \mathrm{IC}(0.43 \pm 0.06$ and $0.39 \pm 0.05 \mathrm{~L}$, respectively) and minute ventilation (31.1 \pm 1.6 and $32.7 \pm 2.2 \mathrm{~L} \cdot \mathrm{min}^{-1}$, respectively) were not significantly different.

Resting hyperinflation helps to explain the limitation of interval exercise. Implementation of interval exercise for rehabilitation should provide important clinical benefits because it prolongs exercise endurance time and allows sustaining higher stable ventilation.

Eur Respir J 2004; 24: 385-390.
*Dept of Pulmonary and Critical Care Medicine, Cardiopulmonary Rehabilitation Centre, Eugenidion Hospital, ${ }^{\#}$ Dept of Physical Education and Sport Science, National and Kapodistrian University of Athens, and "Third Pulmonary Dept, Seismanoglion Hospital, Athens, Greece.

Correspondence: I. Vogiatzis, National and Kapodistrian University of Athens Medical School, Dept of Pulmonary and Critical Care Medicine, Eugenidion Hospital 2nd Floor, 20 Papandiamantopoulou Str 115-28 Ilisia, Athens, Greece.

Fax: 302107242785

E-mail: gianvog@phed.uoa.gr

Keywords: Chronic obstructive pulmonary disease, dynamic hyperinflation, dyspnoea, interval exercise

Received: November 192003

Accepted after revision: May 102004

This work was supported in part by the European Community. Project title: Computer Aided Rehabilitation of Respiratory Disabilities (CARED) FP5 (contract no. QLG5-CT-2002-0893).
Recent studies have demonstrated the benefits of rehabilitative exercise training on exercise capacity, quality of life and utilisation of healthcare resources in patients with chronic obstructive pulmonary disease (COPD) [1]. Most rehabilitation programmes are based on constant-load exercise (CLE) training, consisting of sustained exercise for 30-40 min [1]. Generally, high-intensity training is argued to be needed for the improvement of exercise capacity [2]. Although patients with moderately severe COPD (mean forced expiratory volume in one second (FEV1) $>45 \%$ predicted) can tolerate high levels $(80 \%)$ of their peak tolerance for several minutes $[3,4]$, patients with more severe disease are unable to tolerate such exercise intensities for sufficiently long periods $[5,6]$.

The factors that limit exercise tolerance in these patients are linked with the development of dynamic hyperinflation (DH) and the concurrent mechanical constraints on ventilation that contribute importantly to perceived respiratory discomfort. Secondary to $\mathrm{DH}$ and concomitant high mean intrathoracic pressure, cardiac performance and, hence, supply of oxygenated blood to the malfunctioning peripheral muscles are further compromised $[7,8]$. This contributes to perceived leg discomfort and exercise intolerance. The importance of DH in exercise limitation in patients with advanced COPD arises from studies which demonstrated that alleviation of dyspnoea with acute bronchodilator therapy or oxygen supplementation is due, in part, to decreased operating lung volumes [9-11]. Both interventions improved exercise endurance time (tend), which, nonetheless, remained brief (typically $\sim 8-10 \mathrm{~min}$ ).

Interval exercise (IE) training, which consists of maximalintensity exercise loads on peripheral muscles, has been used by the current authors as an equally effective alternative to CLE in patients with moderately severe COPD (mean FEV1 $45 \%$ pred) [12]. Interestingly, the levels of dyspnoea in the IE group during the training sessions were significantly lower than in the CLE group. On the basis of the well-established mechanistic link between the intensity of dyspnoea and the degree of DH [13], the lower symptoms of dyspnoea during IE might reflect smaller increases in dynamic lung volumes as compared with CLE. However, there are no studies demonstrating the degree to which the behaviour of operating lung volumes during IE influences exercise tolerance in patients with severe COPD. Moreover, as IE consists of a sequence of on and off high-intensity muscular loading events, its tolerability in the context of perceived respiratory and peripheral muscle discomfort is still unknown.

Accordingly, the objectives of the current study were as follows: 1) to determine the range and pattern of change in the operating lung volume components during IE in patients with different degrees of airflow limitation; and 2) to investigate 
whether IE could enable patients with advanced COPD to tolerate high-intensity exercise for sufficiently long periods of time.

\section{Methods}

\section{Subjects}

Subjects included 27 male patients with stable COPD who satisfied the following criteria: 1) postbronchodilator FEV1 $<50 \%$ pred and FEV1/forced vital capacity $<65 \%$ without significant reversibility $(<12 \%$ change of the initial FEV1 value); 2) optimised medical therapy; and 3) no clinical evidence of exercise-limiting cardiovascular or neuromuscular diseases.

\section{Study design}

Patients underwent three symptom-limited exercise tests on different days in the following order: 1) a ramp-incremental test to define the peak work rate (WRmax); 2) a CLE test at a work rate equivalent to $80 \% \mathrm{WRmax}$; and 3) an IE test at a work rate that corresponded to $100 \%$ WRmax with $30 \mathrm{~s}$ work, interspersed with $30 \mathrm{~s}$ of unloaded pedalling. All patients signed an informed consent and the protocol was approved by the current authors' hospital ethics committee.

\section{Pulmonary function tests}

Spirometry and lung diffusion capacity for carbon monoxide ( $D$ L,CO) were performed by a spirometer (Masterlab; Jaeger, Wurzburg, Germany) according to recommended techniques [14], whereas maximum voluntary ventilation (MVV) was directly measured (Vmax 229; Sensor Medics, Anaheim, CA, USA). Arterial blood was drawn by puncture of the radial artery at rest, whilst breathing room air for the analysis of arterial oxygen tension $\left(\mathrm{Pa}, \mathrm{O}_{2}\right)$, carbon dioxide tension $\left(\mathrm{Pa}, \mathrm{CO}_{2}\right)$ and $\mathrm{pH}(\mathrm{ABL} 330$; Radiometer, Copenhagen, Denmark).

\section{Exercise testing}

All tests were performed on an electromagnetically braked cycle ergometer (Ergoline 800; Sensor Medics), with the subjects maintaining a pedalling frequency of $60 \mathrm{rpm}$. Tests were preceded by a 2 -min rest period, followed by $3 \mathrm{~min}$ of unloaded pedalling. The following pulmonary gas exchange and ventilatory variables were recorded breath-by-breath (Vmax 229; Sensor Medics): oxygen uptake $\left(V^{\prime} \mathrm{O}_{2}\right)$, carbon dioxide output $\left(V^{\prime} \mathrm{CO}_{2}\right)$, respiratory exchange ratio, minute ventilation $\left(V^{\prime} \mathrm{E}\right)$, tidal volume $(V \mathrm{~T})$, and breathing frequency. Cardiac frequency $(f \mathrm{C})$ and percentage oxygen saturation measured by pulse oximetry were determined using the $\mathrm{R}-\mathrm{R}$ interval from a 12-lead online electrocardiogram (Marquette Max; Marquette Hellige GmbH, Freiburg, Germany) and a pulse oximeter (Nonin 8600; Nonin Medical, Plymouth, MN, USA), respectively. The modified Borg Scale was used to rate the magnitude of perceived dyspnoea and leg discomfort every 3 min throughout and upon cessation of exercise [15]. During the ramp-incremental test (increments of 5-10 W), the anaerobic threshold (AT) was determined via the $\mathrm{V}$-slope technique [16]. The peak $V^{\prime} \mathrm{O}_{2}$ values were compared with those of JonEs [17].

During exercise tests, changes in operational lung volumes were evaluated from measurements of dynamic inspiratory capacity (IC), assuming that total lung capacity (TLC) remained constant during exercise [18], thus reflecting changes in end-expiratory and end-inspiratory lung volumes (EILV). By subtracting $V \mathrm{~T}$ from the coinciding IC, changes in inspiratory reserve volume (IRV) were calculated. Prior to exercise testing, patients were familiarised with the IC manoeuvre, where they were instructed to make 3-5 maximal efforts according to previously described methods, i.e. "at the end of the next normal expiration, take a deep breath all the way in", followed by verbal encouragement to make a maximal effort before relaxing [18, 19]. Throughout exercise testing, IC was measured every $3 \mathrm{~min}$ and at the end of exercise. During the IE tests, IC measurements were carried out during the 30 -s work phases. Arterial blood for the determination of $\mathrm{Pa}, \mathrm{O}_{2}, \mathrm{~Pa}, \mathrm{CO}_{2}, \mathrm{pH}$, alveolar-arterial oxygen pressure difference, arterial-end tidal carbon dioxide pressure difference and arterial lactate concentration was drawn before and at the end of the tests.

\section{Data analysis}

Data were presented as mean \pm SEM. A statistical significance of 0.05 was used for all analyses, with appropriate Bonferroni corrections for multiple comparisons. During IE, temporally matched (at 30,60 and $90 \%$ of the total exercise time) measurements for $V^{\prime} \mathrm{O}_{2}, f \mathrm{C}$, and $V^{\prime} \mathrm{E}$, corresponding to successive 30-s intervals of work and unloaded pedalling, were analysed by a two-way analysis of variance (ANOVA) with repeated measures and the appropriate post hoc analysis. Similarly, changes in IC and rates of perceived dyspnoea and leg discomfort across the different work phases were assessed by ANOVA with repeated measures. Within and between group comparisons were performed using paired and unpaired t-tests, respectively. Linear regression analysis was performed using the least square method. When this analysis was carried out using IE tend as a dependent variable, the independent variables included the resting pulmonary function and IE measurements. The strongest significant contributors to tend were selected using stepwise multiple regression analysis.

\section{Results}

\section{Patient characteristics}

As shown in table 1, patients were characterised by severe airflow limitation, moderate hypoxaemia and a substantially reduced $D \mathrm{~L}, \mathrm{CO}$ with considerable alveolar ventilation/perfusion inequality, as reflected by the high physiological dead space $(V \mathrm{D}) / V \mathrm{~T}$. Mean resting IC was reduced below normal limits $(<80 \%$ pred) at $1.98 \pm 0.09 \mathrm{~L}$ or $67 \pm 3 \%$ of the predicted normal; predicted normal values for IC were calculated as pred TLC-pred functional residual capacity $[13,18]$. The $95 \%$ confidence interval $(\mathrm{CI})$ for resting IC measurements was \pm 0.20 L or $\pm 6.9 \%$ pred. Exercise capacity was severely compromised because of ventilatory limitation, as peak exercise $V^{\prime} \mathrm{E}$ approached the maximal ventilatory capacity, i.e. mean $V^{\prime} \mathrm{E} / \mathrm{MVV}$ was $90 \pm 3 \%$ (table 2). The latter constitutes one of the criteria when patient effort is usually considered to be maximal [20]. At the limit of tolerance, patients' EILV closely approached their TLC, whereas IRV was reduced to $0.28 \pm 0.03 \mathrm{~L}$ or $4.4 \pm 0.8 \%$ pred TLC. The lowest IRV value that was achieved at the peak of symptom-limited incremental exercise test was defined as minimal IRV [13]. At the limit of tolerance, the change $(\Delta)$ in IC from its resting value amounted to $-0.52 \pm 0.06 \mathrm{~L}$ or $-17.6 \pm 2.0 \%$ pred, which was 
well beyond the $95 \% \mathrm{CI}$ of the resting value. The AT could be identified in 22 out of 27 patients.

Table 1. - Physical and baseline pulmonary function characteristics of patients

\begin{tabular}{|c|c|}
\hline Age yrs & $67 \pm 1$ \\
\hline Height $\mathrm{cm}$ & $170 \pm 1$ \\
\hline Weight $\mathrm{kg}$ & $76 \pm 3$ \\
\hline $\mathrm{BMI} \mathrm{kg} \cdot \mathrm{m}^{-2}$ & $25.8 \pm 0.8$ \\
\hline FEV1 L & $1.14 \pm 0.07$ \\
\hline FEV1 $\%$ pred & $39.7 \pm 2.6$ \\
\hline FVC L & $2.44 \pm 0.13$ \\
\hline FVC $\%$ pred & $65.7 \pm 3.8$ \\
\hline FEV $1 / F V C \%$ pred & $47.3 \pm 2.0$ \\
\hline$D \mathrm{~L}, \mathrm{CO} \%$ pred & $52.2 \pm 3.6$ \\
\hline$P \mathrm{a}, \mathrm{O}_{2} \mathrm{mmHg}$ & $71.0 \pm 1.7$ \\
\hline $\mathrm{Pa}_{\mathrm{a}} \mathrm{CO}_{2} \mathrm{mmHg}$ & $42.6 \pm 1.0$ \\
\hline $\mathrm{pH}$ & $7.42 \pm 0.04$ \\
\hline Arterial lactate conc. $\mathrm{mMol} \cdot \mathrm{L}^{-1}$ & $1.3 \pm 0.1$ \\
\hline$V \mathrm{D} \mathrm{L}$ & $0.36 \pm 0.03$ \\
\hline$V \mathrm{D} / V \mathrm{~T} \%$ & $44 \pm 2$ \\
\hline IC L & $1.98 \pm 0.09$ \\
\hline IC $\%$ pred & $67 \pm 3$ \\
\hline $\mathrm{MVV} \mathrm{L} \cdot \min ^{-1}$ & $46 \pm 4$ \\
\hline
\end{tabular}

Data are presented as mean \pm SEM. BMI: body mass index; FEV1: forced expiratory volume in one second; \% pred: percentage of predicted value; FVC: forced vital capacity; $D$ L,CO: transfer factor for carbon monoxide; $\mathrm{Pa}, \mathrm{O}_{2}$ : arterial oxygen tension; $\mathrm{Pa}_{\mathrm{a}} \mathrm{CO}_{2}$ : arterial carbon dioxide tension; conc.: concentration; $V \mathrm{D}$ : physiological dead space; VT: tidal volume; IC: inspiratory capacity; MVV: maximum voluntary ventilation. $1 \mathrm{mmHg}=0.1 \mathrm{kPa}$.

Table 2. - Peak responses to the ramp-incremental exercise test

\begin{tabular}{|c|c|}
\hline WR W & $76 \pm 5$ \\
\hline WR \% pred & $51 \pm 3$ \\
\hline$V^{\prime} \mathrm{O}_{2} \mathrm{~L} \cdot \mathrm{min}^{-1}$ & $1.09 \pm 0.07$ \\
\hline$V^{\prime} \mathrm{O}_{2} \%$ pred & $54 \pm 3$ \\
\hline$V^{\prime} \mathrm{CO}_{2} \mathrm{~L} \cdot \min ^{-1}$ & $1.10 \pm 0.08$ \\
\hline $\mathrm{R}$ & $1.01 \pm 0.03$ \\
\hline$V^{\prime} \mathrm{E} \mathrm{L} \cdot \mathrm{min}^{-1}$ & $40.0 \pm 2.1$ \\
\hline$V^{\prime} \mathrm{E} / \mathrm{MVV} \%$ & $90 \pm 3$ \\
\hline $\mathrm{BR} \mathrm{L} \cdot \mathrm{min}^{-1}$ & $6.4 \pm 3.9$ \\
\hline$V^{\prime} \mathrm{E} / V^{\prime} \mathrm{O}_{2}$ & $37.9 \pm 1.4$ \\
\hline$V^{\prime} \mathrm{E} / V^{\prime} \mathrm{CO}_{2}$ & $38.6 \pm 1.4$ \\
\hline$V \mathrm{~T} \mathrm{~L}$ & $1.18 \pm 0.06$ \\
\hline$f$ breaths $\cdot \min ^{-1}$ & $34 \pm 1$ \\
\hline$f \mathrm{C}$ beats $\cdot \min ^{-1}$ & $126 \pm 4$ \\
\hline$f \mathrm{C} \%$ pred & $83 \pm 2$ \\
\hline AT $\mathrm{L} \cdot \mathrm{min}^{-1}$ & $0.74 \pm 0.04$ \\
\hline $\mathrm{SP}, \mathrm{O}_{2} \%$ & $90 \pm 2$ \\
\hline $\mathrm{IC} \mathrm{L}$ & $1.46 \pm 0.10$ \\
\hline IC $\%$ pred & $49.5 \pm 3.1$ \\
\hline$\Delta \mathrm{IC}$ from rest $\mathrm{L}$ & $-0.52 \pm 0.06$ \\
\hline$\Delta \mathrm{IC}$ from rest $\%$ pred & $-17.6 \pm 2.0$ \\
\hline$V \mathrm{~T} / \mathrm{IC} \%$ & $80.8 \pm 1.7$ \\
\hline IRV L & $0.28 \pm 0.03$ \\
\hline IRV $\%$ pred TLC & $4.4 \pm 0.8$ \\
\hline Dyspnoea Borg & $4.7 \pm 0.3$ \\
\hline Leg discomfort Borg & $5.0 \pm 0.3$ \\
\hline
\end{tabular}

Data are presented as mean \pm SEM. WR: work rate in watts; $\%$ pred: percentage of predicted value; $V^{\prime} \mathrm{O}_{2}$ : oxygen uptake; $V^{\prime} \mathrm{CO}_{2}$ : carbon dioxide output; R: respiratory exchange ratio; $V^{\prime} \mathrm{E}$ : minute ventilation; MVV: maximum voluntary ventilation; BR: breathing reserve; $V^{\prime} \mathrm{E} /$ $V^{\prime} \mathrm{O}_{2}$ : ventilatory equivalent for $V^{\prime} \mathrm{O}_{2} ; V^{\prime} \mathrm{E} / V^{\prime} \mathrm{CO}_{2}$ : ventilatory equivalent for $V^{\prime} \mathrm{CO}_{2} ; V \mathrm{~T}$ : tidal volume; $f$ : breathing frequency; $f \mathrm{C}$ : cardiac frequency; AT: anaerobic threshold (identified in 22 out of 27 patients); $S \mathrm{P}, \mathrm{O}_{2}$ : arterial oxygen saturation measured by pulse oximetry; IC: inspiratory capacity; $\triangle \mathrm{IC}$ from rest: change in IC from rest; IRV: inspiratory reserve volume; TLC: total lung capacity.

\section{Physiological responses during interval exercise}

Average tend $(32.7 \pm 3.0 \mathrm{~min})$ was significantly longer compared with the mean tend recorded for CLE (10.3 $\pm 1.6 \mathrm{~min})$ in the present study (table 3 ). The time course of changes in $V^{\prime} \mathrm{O}_{2}, V^{\prime} \mathrm{CO}_{2}, V^{\prime} \mathrm{E}$ and IC during the IE protocol in two representative patients is shown in figure 1. ANOVA with repeated measures revealed that measurements of $V^{\prime} \mathrm{O}_{2}$, $V^{\prime} \mathrm{CO}_{2}$ and $V^{\prime} \mathrm{E}$ did not differ significantly between work and unloaded pedalling phases in either of the temporally matched measurements that were taken at time points corresponding to 30,60 and $90 \%$ of the total duration during IE (table 4). Similarly, IC did not differ significantly across the different work phases (table 4).

\section{Measurements of operational lung volumes}

At the end of symptom-limited IE, IC was significantly reduced from its baseline value by $0.39 \pm 0.05 \mathrm{~L}$; this corresponded to a mean $\Delta \mathrm{IC}$ of $-20 \pm 4 \%$ or $-13 \pm 2 \%$ pred, which was well beyond the $95 \%$ CI for IC. IE produced significant volume constraints on $V \mathrm{~T}$ expansion, indicated by the higher reduction in IRV in the current authors' COPD population than in normal populations (table 3) [13]. However, at the end of IE, the IRV value $(8.9 \pm 0.8 \%$ pred TLC) was significantly higher compared to the minimal IRV that was recorded at the limit of tolerance during the rampincremental and CLE tests (tables 2 and 3).

Table 3.-Responses to constant-load exercise (CLE) and interval exercise (IE) protocols

\begin{tabular}{|c|c|c|}
\hline & End of CLE & End of IE \\
\hline Exercise time min & $10.3 \pm 1.6$ & $32.7 \pm 3.0^{\#}$ \\
\hline$V^{\prime} \mathrm{O}_{2} \mathrm{~L} \cdot \mathrm{min}^{-1}$ & $0.94 \pm 0.07$ & $0.81 \pm 0.05^{\#}$ \\
\hline$V^{\prime} \mathrm{CO}_{2} \mathrm{~L} \cdot \mathrm{min}^{-1}$ & $0.95 \pm 0.07$ & $0.75 \pm 0.04^{\#}$ \\
\hline $\mathrm{R}$ & $1.00 \pm 0.02$ & $0.95 \pm 0.02^{\#}$ \\
\hline$V^{\prime} \mathrm{E} \mathrm{L} \cdot \mathrm{min}^{-1}$ & $38.4 \pm 2.2$ & $33.1 \pm 1.5^{\#}$ \\
\hline$V^{\prime} \mathrm{E} / \mathrm{MVV} \%$ & $85 \pm 3$ & $76 \pm 4^{\#}$ \\
\hline$V \mathrm{~T} \mathrm{~L}$ & $1.14 \pm 0.06$ & $1.01 \pm 0.05^{\#}$ \\
\hline$f$ breaths $\cdot \min ^{-1}$ & $34 \pm 1$ & $33 \pm 1$ \\
\hline$f \mathrm{C}$ beats $\cdot \min ^{-1}$ & $128 \pm 5$ & $123 \pm 5$ \\
\hline IC L & $1.53 \pm 0.08$ & $1.59 \pm 0.08^{\circ}$ \\
\hline IC $\%$ pred & $51.9 \pm 3.0$ & $53.9 \pm 2.6^{\bullet}$ \\
\hline$\Delta \mathrm{IC} \mathrm{L}$ & $-0.45 \pm 0.05$ & $-0.39 \pm 0.05^{\oplus}$ \\
\hline$\Delta \mathrm{IC} \%$ pred & $-15.2 \pm 2.4$ & $-13.3 \pm 1.5^{\oplus}$ \\
\hline$V \mathrm{~T} / \mathrm{IC} \%$ & $75.2 \pm 1.7$ & 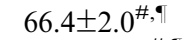 \\
\hline IRV L & $0.38 \pm 0.03$ & $0.58 \pm 0.06^{\#, 9}$ \\
\hline IRV $\%$ pred TLC & $5.9 \pm 0.5$ & $8.9 \pm 0.8^{\#, \oplus}$ \\
\hline $\mathrm{Pa}, \mathrm{O}_{2} \mathrm{mmHg}$ & $66.7 \pm 2.6$ & $68.7 \pm 2.5$ \\
\hline $\mathrm{Pa}, \mathrm{CO}_{2} \mathrm{mmHg}$ & $45.4 \pm 1.2$ & $44.0 \pm 1.3^{\#,+}$ \\
\hline $\mathrm{pH}$ & $7.34 \pm 0.07$ & $7.38 \pm 0.06^{\#,+}$ \\
\hline$V \mathrm{D} \mathrm{L}$ & $0.53 \pm 0.02$ & $0.48 \pm 0.02^{\#,+}$ \\
\hline$V \mathrm{D} / V \mathrm{~T} \%$ & $46 \pm 6$ & $47 \pm 8^{+}$ \\
\hline Arterial lactate conc. $\mathrm{mMol} \cdot \mathrm{L}^{-1}$ & $5.81 \pm 0.41$ & $3.89 \pm 0.36^{\#,+}$ \\
\hline Dyspnoea Borg & $4.8 \pm 0.3$ & $4.3 \pm 0.3$ \\
\hline Leg discomfort Borg & $4.9 \pm 0.4$ & $4.6 \pm 0.5$ \\
\hline
\end{tabular}

Data are presented as mean \pm SEM. $V^{\prime} \mathrm{O}_{2}$ : oxygen uptake; $V^{\prime} \mathrm{CO}_{2}$ : carbon dioxide output; R: respiratory exchange ratio; $V^{\prime} \mathrm{E}$ : minute ventilation; MVV: maximum voluntary ventilation; $V \mathrm{~T}$ : tidal volume; $f$ : breathing frequency; $f \mathrm{C}$ : cardiac frequency; IC: inspiratory capacity; $\Delta \mathrm{IC}$ : change in IC from rest; IRV: inspiratory reserve volume; IRV \% pred TLC: IRV as a percentage of the predicted value for total lung capacity; $\mathrm{Pa}, \mathrm{O}_{2}$ : arterial oxygen tension; $\mathrm{Pa}, \mathrm{CO}_{2}$ : arterial carbon dioxide tension; $V \mathrm{D}$ : physiological dead space; conc.: concentration. \#: significant differences between IE and CLE; ${ }^{\prime}$ : significant differences between the incremental exercise test (table 2) and the terminal point of the IE test; ${ }^{+}$: significant differences between baseline and the terminal point of the IE test. $1 \mathrm{mmHg}=0.1 \mathrm{kPa}$. 

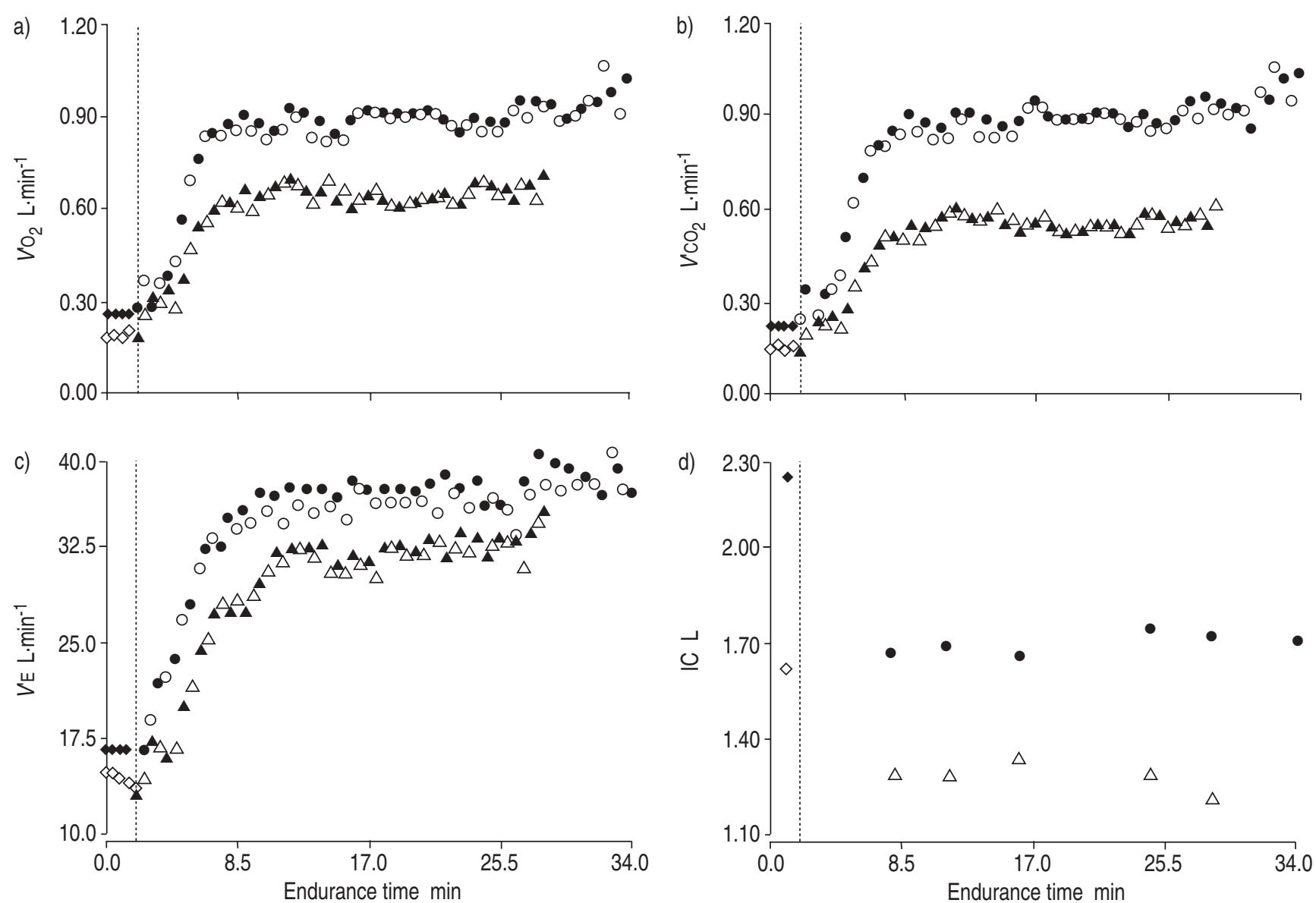

Fig. 1. - Time course of a) oxygen uptake $\left(V^{\prime} \mathrm{O}_{2}\right)$, b) carbon dioxide output $\left(V^{\prime} \mathrm{CO}_{2}\right)$, c) minute ventilation $\left(V^{\prime} \mathrm{E}\right)$ and d) inspiratory capacity $(\mathrm{IC})$ in a patient with $\mathrm{CO}_{2}$ retention (arterial carbon dioxide tension $>45 \mathrm{mmHg} ; \boldsymbol{\Delta}$ and $\triangle$ ) and a patient without $\mathrm{CO}_{2}$ retention $(\bullet$ and $\bigcirc$ ) during the interval exercise protocol. Gas exchange values are 30-s average measurements of baseline $(\diamond$ and $\diamond)$, unloaded pedalling phases (open symbols) and interval exercise loaded phases (closed symbols). Dashed lines represent the onset of interval exercise.

\section{Correlates of interval exercise tolerance}

The tend $(32.7 \pm 3.0 \mathrm{~min})$ correlated with resting IC, expressed as $\%$ pred normal $(\mathrm{r}=0.46, \mathrm{p}<0.01)$, and, additionally, with the $P \mathrm{a}, \mathrm{CO}_{2}(\mathrm{r}=-0.44, \mathrm{p}<0.02)$ and the $V \mathrm{~T}(\mathrm{r}=0.43$, $\mathrm{p}<0.01$ ), both recorded at the limit of tolerance during IE. Using stepwise multiple regression analysis, tend was best described by the combination of $V \mathrm{~T}$ and $\mathrm{IC} \%$ pred recorded at the terminal point of IE $(r=0.55, p<0.02)$. In turn, IC $\%$ pred and $V \mathrm{~T}$ at the limit of tolerance during IE correlated strongly with the end exercise $P \mathrm{a}, \mathrm{CO}_{2}(\mathrm{r}=-0.64, \mathrm{p}<0.0001$ and $\mathrm{r}=-0.58, \mathrm{p}<0.001$, respectively) and $P \mathrm{a}, \mathrm{O}_{2}(\mathrm{r}=0.55, \mathrm{p}<0.001$ and $\mathrm{r}=0.44, \mathrm{p}<0.02$, respectively).

\section{Exertional symptoms}

During IE, symptoms of dyspnoea and leg discomfort increased significantly across the temporally matched time points (table 4). At the limit of tolerance, symptoms were not significantly different compared with those recorded at the end of the incremental (table 2) and the CLE (table 3) protocols.

Table 4.-Responses to interval exercise at temporally matched time points between work and unloaded pedalling phases

\begin{tabular}{|c|c|c|c|c|c|c|}
\hline & \multicolumn{2}{|c|}{$30 \%$ total time } & \multicolumn{2}{|c|}{$60 \%$ total time } & \multicolumn{2}{|c|}{$90 \%$ total time } \\
\hline & Work phase & Unloaded phase & Work phase & Unloaded phase & Work phase & Unloaded phase \\
\hline$V^{\prime} \mathrm{O}_{2} \mathrm{~L} \cdot \min ^{-1}$ & $0.74 \pm 0.04$ & $0.74 \pm 0.04$ & $0.77 \pm 0.05$ & $0.77 \pm 0.05$ & $0.78 \pm 0.04$ & $0.78 \pm 0.05$ \\
\hline$V^{\prime} \mathrm{E} \mathrm{L} \cdot \mathrm{min}^{-1}$ & $31.1 \pm 1.6$ & $31.0 \pm 1.5$ & $32.0 \pm 1.8$ & $32.0 \pm 2.0$ & $32.7 \pm 2.2$ & $32.3 \pm 2.0$ \\
\hline$f \mathrm{C}$ beats $\cdot \min ^{-1}$ & $113 \pm 5$ & $114 \pm 6$ & $118 \pm 5^{\#}$ & $118 \pm 7$ & $120 \pm 4^{\#}$ & $120 \pm 5$ \\
\hline IC L & $1.55 \pm 0.08$ & & $1.61 \pm 0.08$ & & $1.59 \pm 0.08$ & \\
\hline Dyspnoea Borg & $2.9 \pm 0.2$ & & $3.3 \pm 0.3^{\#}$ & & $4.3 \pm 0.4^{\#}$ & \\
\hline Leg discomfort Borg & $3.1 \pm 0.3$ & & $3.6 \pm 0.4^{\#}$ & & $4.6 \pm 0.5^{\#}$ & \\
\hline
\end{tabular}

Data are presented as mean \pm SEM. $V^{\prime} \mathrm{O}_{2}$ : oxygen uptake; $V^{\prime} \mathrm{E}$ : minute ventilation; $f \mathrm{C}$ : cardiac frequency; IC: inspiratory capacity. ${ }^{\#}$ : significant differences between the work phases of the temporally matched time points recorded at $30 \%$ of the total exercise time and those recorded at 60 and $90 \%$ of the total exercise time. 
Arterial blood gases, arterial lactate concentration and anaerobic threshold

At the limit of tolerance during IE, there was a significant increase in $\mathrm{Pa}, \mathrm{CO}_{2}$ and $V \mathrm{D}$ from rest (table 3 ). Arterial lactate concentration was significantly higher, and arterial $\mathrm{pH}$ was lower, at the end of IE compared to baseline. Compared to the terminal point of CLE (table 3), arterial lactate concentration, $\mathrm{Pa}, \mathrm{CO}_{2}$ and $V \mathrm{D}$ were significantly lower, and arterial $\mathrm{pH}$ was higher, at the end of IE. $V^{\prime} \mathrm{O}_{2}$ at the AT $\left(0.74 \mathrm{~L} \cdot \mathrm{min}^{-1}\right)$ was not significantly different to the mean $V^{\prime} \mathrm{O}_{2}$ $\left(0.78 \mathrm{~L} \cdot \mathrm{min}^{-1}\right)$ sustained during IE in the 22 patients whose AT was identified.

\section{Discussion}

The principal aim of the present study was to evaluate the influence of $\mathrm{DH}$ on IE tolerance in patients with severe airflow limitation. Additionally, it was examined whether IE would enable such patients to tolerate high-intensity exercise for a sufficient time to achieve physiological training effects. The main findings were as follows: 1) the total exercise duration during IE was significantly greater than at CLE; 2) IE was associated with stable metabolic and ventilatory responses as the mean exercise $V^{\prime} \mathrm{O}_{2}$ was slightly above the patients' AT; this, in turn, allowed patients to exercise for a prolonged period of time before symptoms limited the exercise endurance capacity; and 3) symptom-limited IE tend correlated with resting hyperinflation.

Historically, the rationale for IE training has been the ability to impose very high power outputs to peripheral muscles without overloading the cardiorespiratory capacity [21]. Classical studies [21] have shown that the metabolic response during IE is very similar to continuous moderate exercise and, thus, is associated with a stable pattern of cardiorespiratory responses and low lactate concentration in the muscle throughout the relatively long exercise and recovery periods. This was shown in the present study by the fact that IE was associated with a relatively stable metabolic and ventilatory response, i.e. $V^{\prime} \mathrm{O}_{2}$ and $V^{\prime} \mathrm{E}$ changed very little throughout the exercise and unloaded pedalling phases, and corresponded to values typically seen during CLE at markedly lower intensity $(50-70 \% \mathrm{WR} \max )$ in patients with similar degrees of airflow limitation [22, 23]. Interestingly, in spite of the fact that mean symptom-limited $V^{\prime} \mathrm{O}_{2}$ was slightly above the patients' AT, the tend was relatively long, which was not the case during CLE (table 3) above the AT [3]. Moreover, the small increase in arterial lactate concentration to the terminal point of exercise further supports the notion that IE closely resembled steady-state exercise. The capacity to reload myoglobin stores during the recovery phases, allowing a more oxidative degradation of glycogen and, hence, a partially reduced demand, has been proposed as the principal mechanism for the slowed glycolysis observed during IE [21]. As lactic acidosis puts particular stress on the ventilatory system, the small increase in arterial lactate concentration observed during IE as compared to CLE (table 3) appeared to be beneficial to the COPD patients by reducing some of the acid stimulus to breathe [4], thereby maintaining ventilation and dyspnoea at sustainable levels for a prolonged period of time.

This is the first study to document DH during IE in COPD patients. Patients were dynamically hyperinflated throughout the IE test, as shown by the significant reduction in IC from baseline, which averaged $\sim 0.40 \mathrm{~L}$ or $13 \%$ of IC pred normal. This is similar to the values reported by $\mathrm{O}^{\prime}$ DONNELL and colleagues $[9,22,23]$ during CLE in patients with a comparable degree of severity and close to the value $(0.45 \mathrm{~L})$ recorded during the CLE protocol in the present study (table 3). An interesting feature of the present study was that IC did not change significantly throughout IE in contrast to CLE $[9,23]$. In spite of DH, the current authors' patients were able to sustain bouts of maximal-intensity loads for a period of time that was several times longer compared to the present and other CLE protocols [3, 4, 9, 10, 11, 22, 23]. Whilst facing steady metabolic and ventilatory demands during IE, patients were able to sustain levels of pulmonary ventilation averaging $76 \% \mathrm{MVV}$ at the terminal point of exercise for a prolonged period. This is further supported by the finding that, even at the limit of tolerance, the patients' IRV was significantly higher $(p=0.01)$ than the IRV attained at the end of the incremental and CLE tests $(0.58 \pm 0.06 \mathrm{~L}$ versus $0.28 \pm 0.03 \mathrm{~L}$ and $0.38 \pm 0.03 \mathrm{~L}$, respectively).

In agreement with previous studies [6, 9, 10, 23, 24], it was found that, at the limit of interval exercise tolerance, IC and $V \mathrm{~T}$ were the most important contributors to tend. This is not surprising as the dynamic IC has been shown to reflect the operating limits for $V \mathrm{~T}$ expansion during incremental [13, 24] and CLE protocols $[9,10,23]$. The $\mathrm{Pa}_{\mathrm{a}} \mathrm{CO}_{2}$ also emerged as a significant contributory variable to endurance capacity, confirming previous findings that the propensity to develop $\mathrm{CO}_{2}$ retention during exercise reflects ventilatory constraints due to prolonged hyperinflation [6-26]. In turn, IC \% pred normal and the curtailed $V \mathrm{~T}$ response at the limit of IE tolerance were strongly correlated with changes in $\mathrm{Pa}, \mathrm{CO}_{2}$ and $\mathrm{Pa}, \mathrm{O}_{2}$ from baseline, thus reflecting the effects of worsening alveolar ventilation/perfusion inequalities.

In summary, it has been shown that, in severely disabled chronic obstructive pulmonary disease patients, interval exercise is associated with stable metabolic demands, minute ventilation and rates of dynamic hyperinflation, and that the total exercise duration is much greater than at constant-load exercise. Hence, the application of this method in the rehabilitation setting has the potential to convey important clinical benefits, as it allows the application of intense loads on peripheral muscles for sufficiently long periods of time in order to obtain the desired physiological training effects. Interval exercise may, therefore, provide a good alternative to constant-load rehabilitative exercise training in order to improve compliance with high-intensity exercise and, thus, the effectiveness of this treatment.

\section{References}

1. ACCP/AACVPR Pulmonary Rehabilitation Guidelines Panel, American College of Chest Physicians, American Association of Cardiovascular and Pulmonary Rehabilitation. Pulmonary rehabilitation: joint ACCP/AACVRR evidence based guidelines. Chest 1997; 112: 1363-1396.

2. Casaburi R. Special considerations for exercise training. In: ACSM resource manual for guidelines for exercise testing and prescription. 4th Edn. ACSM, 2001; pp. 346-352.

3. Neder A, Jones PW, Nery LE, Whipp BJ. Determinants of the exercise endurance capacity in patients with chronic obstructive pulmonary disease. Am J Respir Crit Care Med 2000; 162: 497-504.

4. Casaburi R, Patessio A, Ioli F, Zanaboni S, Donner CF, Wasserman K. Reductions in lactic acidosis and ventilation as a result of exercise training in patients with obstructive lung disease. Am Rev Respir Dis 1991; 143: 9-18.

5. Maltais F, LeBlank P, Jobin J, et al. Intensity of training and physiologic adaptations in patients with chronic obstructive pulmonary disease. Am J Respir Crit Care Med 1997; 155: 555-561.

6. O'Donnell DE, D'Arsigny, Fitzpatrick M, Webb KA. Exercise hypercapnia in advanced chronic obstructive 
pulmonary disease. The role of lung hyperinflation. $\mathrm{Am}$ J Respir Crit Care Med 2002; 166: 663-668.

7. A statement of the American Thoracic Society and the European Respiratory Society. Skeletal muscle dysfunction in chronic obstructive pulmonary disease. Am J Respir Crit Care Med 1999; 159: S1-S40.

8. Aliverti A, Maklem PT. How and why exercise is impaired in COPD. Respiration 2001; 68: 229-239.

9. O'Donnell DE, Lam M, Webb KA. Spirometric correlates of improvement in exercise performance after anticholinergic therapy in chronic obstructive pulmonary disease. $\mathrm{Am}$ J Respir Crit Care Med 1998; 160: 542-549.

10. O'Donnell DE, D'Arsigny C, Webb KA. Effects of hyperoxia on ventilatory limitation during exercise in advanced chronic obstructive pulmonary disease. Am J Respir Crit Care Med 2001; 163: 892-898.

11. Somfay A, Porszasz SM, Lee SM, Casaburi R. Doseresponse effect of oxygen on hyperinflation and exercise endurance in nonhypoxaemic COPD patients. Eur Respir $J$ 2001; 18: 77-84.

12. Vogiatzis I, Nanas $\mathrm{S}$, Roussos C. Interval training as an alternative modality to continuous exercise in patients with COPD. Eur Respir J 2002; 20: 12-19.

13. O'Donnell DE, Revill SM, Webb KA. Dynamic hyperinflation and exercise intolerance in chronic obstructive pulmonary disease. Am J Respir Crit Care Med 2001; 164: 770-777.

14. Gardner RM, Hankinson JL, Clausen JL, Crapo RO, Johnson Jr RO, Epler GR. American Thoracic Society standardization of spirometry: 1987 update. Am Rev Respir Dis 1987; 136: 1285-1298.

15. Borg GA. Psychophysical bases of perceived exertion. Med Sci Sports Exerc 1982; 14: 377-381.

16. Sue DY, Wasserman K, Moricca RB, Casaburi R. Metabolic acidosis during exercise in patients with chronic obstructive pulmonary disease. Chest 1988; 94: 931-938.
17. Jones NJ. Clinical exercise testing. 3rd Edn. Philadelphia WB Saunders Company, 1988; pp. 243-247.

18. Yan S, Kaminski D, Sliwinsky P. Reliability of inspiratory capacity for estimating end-expiratory lung volume changes during exercise in patients with chronic obstructive pulmonary disease. Am J Respir Crit Care Med 1997; 156: $55-59$.

19. O'Donnell DE, Webb KA. Exertional breathlessness in patients with chronic airflow limitation: the role of lung hyperinflation. Am J Respir Crit Care Med 1993; 148: 13511357.

20. Joint ATS/ACCP statement on cardiopulmonary exercise testing. Assessment of patient effort. Am J Respir Crit Care Med 2003; 167: 211-277.

21. Astrand PO, Rodahl K. Physical training. In: Astrand PO, Rodahl K, eds. Textbook of work physiology. New York, McGraw-Hill, 1986; pp. 412-476.

22. O'Donnell DE, McGuire M, Samis L, Webb KA. General exercise training improves ventilatory and peripheral muscle strength and endurance in chronic airflow limitation. $\mathrm{Am}$ J Respir Crit Care Med 1998; 157: 1489-1497.

23. O'Donnell DE, Lam M, Webb KA. Measurement of symptoms, lung hyperinflation and endurance during exercise in chronic obstructive pulmonary disease. Am J Respir Crit Care Med 1998; 158: 1557-1565.

24. Diaz O, Villafrance $\mathrm{C}$, Ghezzo $\mathrm{H}$, et al. Breathing pattern and gas exchange at peak exercise in COPD patients with and without tidal flow limitation at rest. Eur Respir $J$ 2001; 17: $1120-1127$.

25. Cloosterman SG, Hofland ID, van Schayck CP, Folgering HT. Exertional dyspnoea in patients with airway obstruction, with and without $\mathrm{CO}_{2}$ retention. Thorax 1998; 53: 768-774.

26. Diaz O, Villafrance C, Ghezzo $\mathrm{H}$, et al. Role of inspiratory capacity on exercise tolerance in COPD patients with and without tidal flow limitation at rest. Eur Respir J 2000; 16: 269-275. 